\title{
The relationship between difference and ratio and a proposal: Equivalence of temperature and time, and the first spontaneous symmetry breaking
}

Peter Strazewski

\begin{abstract}
The mathematical form of the energy-entropy relationship is that of the relationship between the difference and the ratio of any two 'entities' $x$ and $y$ creating a geometrical 3D projection $x=y \cdot z$, i.e., a surface of the shape of a hyperbolic paraboloid being a particular form of a quadric. The significance of this relationship is discussed here in a realm beyond thermodynamics. Somewhat intuitively, yet still in strict mathematical analogy to the relation between its most fundamental state variables, I propose for any isolated universe the cosmological state variables, absolute temperature and absolute time, to be equivalent much in the same way as energy and entropy are equivalent for an isolated ensemble of similar objects. According to this principle, the cosmological constant is inversely proportional to the squared product of absolute time and absolute temperature. The spontaneous symmetry breaking into a time component and a temperature component of the universe takes place when the first de facto irreversible movement occurs owing to a growing accessed total volume.
\end{abstract}

\section{The relationship between difference and ratio in mathematics and physics}

In mathematics the concept of 'a group' is used to formulate, in a most general way, any kind of mathematical operation, for example, addition, multiplication or rotation. Generally, a mathematical group consists of 'elements' $a, b, c, \ldots$ and an instruction that attributes to each pair of elements, say $a$ and $b$, a new element $a \circ b$ in such a way that three group axioms G1, G2 and G3 strictly apply. Abelian groups are commutative for which group axiom G4 applies as well:

For any three elements $a, b$ and $c$ associativity applies :

$(a \circ b) \circ c=a \circ(b \circ c)$

For any element $a$ there is an identity element $e$ :

$e \circ a=a \circ e=a$

Correspondence: strazewski@univ-lyon1.fr

Laboratoire de Synthèse de Biomolécules, Institut de Chimie et Biochimie Moléculaires et Supramoléculaires (CNRS UMR 5246), Université Claude Bernard Lyon 1, Université de Lyon, 43 bvd du 11 novembre 1918,

F - 69622, Villeurbanne, France

The mathematical operation of addition in conjunction with elements that are integers, $\{a, b, c, \ldots\} \in \mathbb{Z}$, is an abelian group. To specify, replace the above general formalism with a specific one, i.e., replace $\circ$ with,$+ e$ with 0 , and $a^{-1}$ with $-a$. The mathematical operation of multiplication in conjunction with elements that are positive real numbers, $\{a, b, c, \ldots\} \in \mathbb{R}^{+}$, is also an abelian group: $\circ:=\cdot, e:=1$, and $a^{-1}:=1 / a$. Note that, whereas the addition of real numbers is an abelian group, axiom G3 usually does not apply to the multiplication of positive integers $\{a, b, c, \ldots\} \in \mathbb{Z}^{+}$because generally $\left\{a^{-1}, b^{-1}, c^{-1}, \ldots\right\} \notin \mathbb{Z}$. Most commonly, mathematical groups are used to describe geometrical symmetries; the vast majority of mathematical groups are non-abelian (axiom G4 does not apply), in particular, when the parameter space is higher than 2D (twodimensional). 
In physics and physical cosmology this formalism is extremely useful for the description of spontaneous symmetry breaking into different fundamental forces through the application of gauge theories, such as in Quantum Electrodynamics (QED) or Quantum Chromodynamics (QCD) being the basis of the Standard Model. A gauge theory is a type of field theory in which the Lagrangian is invariant under a certain continuous group of local transformations. The Lagrangian of a dynamical system is a function that summarises the dynamics of the system. Common to all these theories is the utilization of Legendre transformations. The Legendre transformation can be generalized to the Legendre-Fenchel transformation. It is commonly used in thermodynamics and in the Hamiltonian formulation of classical mechanics, for example, to describe various thermodynamic potentials in classical thermodynamics, to derive partition functions from a state equation in statistical thermodynamics, to link Lagrange (classical) mechanics with Hamiltonian (quantum) mechanics, to formulate QED and QCD, and more. In a thermodynamic context the linking of energy with entropy through a quantified similarity argument, as described in group thermodynamics [1], may be viewed as a Legendre transformation on a group, rather than individual, level.

Consider a mathematically general relationship between difference and ratio (quotient) being the operational results obtained from, respectively, the substraction and division of the same elements, say, $x$ and $y$ (eqns. 1 and 2):

$$
u=x-v \cdot y
$$

$$
x / y=v \cdot x /(x-u)
$$

Interpret $x$ and $y$ as any two parameters that are needed to define the characteristics of some state and $v$ as a mathematically independent variable. For example, $\{x, y\}$ may be the coordinates of a point in 2D space and $v$ a force acting on one coordinate (in one direction) but not the other. If $x$ were to represent the distance between two objects in Euclidian 3D space, difference $u$ would describe the movement of these objects through spacetime according to Einstein's special relativity $\mathrm{d} s^{2}=\mathrm{d} x_{\mathrm{i}}^{2}-\mathrm{c}^{2} \cdot \mathrm{d} t^{2}$ where $\mathrm{d}=$ differential, $s=$ distance in spacetime, $x_{\mathrm{i}}=$ spatial Cartesian coordinates $\{x, y, z\}, \mathrm{c}=$ vacuum speed of light, and $t=$ time. Difference $u$ could also represent the incremental change $\Delta$ in free energy upon transformation of one (metastable) macrostate into another, as expressed in the GibbsHelmholtz equation $\Delta G_{\mathrm{T}}=\Delta H-T \cdot \Delta S$, where $\Delta G_{\mathrm{T}}=$ Gibbs free energy change at temperature $T, \Delta H=$ energy change at constant pressure (enthalpy change), and $\Delta S=$ entropy change. Equation 2 describes in a generalised fashion the relationship between difference $u$ and ratio $x / y$ as depicted in Figure 1. Note that $v$ is the root of the function in equation 2 .

To analyse more readily, a set of $2 \mathrm{D}$ projections of the same function is depicted in Figure 2 where $u$ is sampled over a representative range of numerical values for $x$ and $y$. Note that, trivially, $x / y$ requires for $v>0$ the root to lie in the quadrants where both $x$ and $y$ have the same sign (right half of the 2D plot), whereas a negative value for $v$ places the root in the quadrants of opposite signs for $x$ and $y$ (reflection of the hyperbles through $x / y=0$, not shown).

In special relativity the relationship between difference and ratio immediately visualises how the shape of squared distance differentials in spacetime $\mathrm{d} s^{2}$ (ordinate in the above $2 \mathrm{D}$ plot) relates to the ratio of squared spatial distance differentials over squared time differentials $\mathrm{d} x_{\mathrm{i}}^{2} / \mathrm{d} t^{2}$ for $v=\mathrm{c}^{2}$ (abscissa). In thermodynamics the above relationship shows how changes in Gibbs free energy $\Delta G_{\mathrm{T}}$ relate to the temperature at which $\Delta G$ vanishes, $T=\Delta H / \Delta S$ for $\Delta C_{\mathrm{p}}=0$, or in a differential form for infinitesimal state changes: $\mathrm{d} G_{\mathrm{T}}$ vs. $T=\mathrm{d} H / \mathrm{d} S$. It was shown that the $\Delta H$ and $\Delta S$ values of similar objects at their respective equilibrium temperatures are all linearly related to their corresponding $\Delta G_{\mathrm{T}}$ values [1]. These realised experimental values of similar objects gather linearly, in the above plot, close to the root of the function at $\{x-v \cdot y=0, x / y=v\}$, i.e., close to the saddle point. Physics imposes positive $v$ values in both special-relativity and thermodynamics, since there is no immediate physical meaning for a negative speed of light (reaching in vacuo a maximum constant positive value c) nor negative absolute temperature, both, in accord with the Second Law.

General and special relativity describe the shape of and the dynamics within a spacetime where the objects may or may not move in a reversible fashion. The main focus in relativity theory is not reversibility but rather to describe objects (particles) and their 'directed' movements at up to relativistic speeds along geodesics in spacetime being shaped by gravitational fields. Thermodynamics describes the energetics of dynamic systems that contain multi-component objects ('macrostates') able to adopt, essentially reversibly, multiple isoergonic (degenerate) 'microstates'. Both theories are, in terms of physics, quite different from one another. The former is based on Einstein's mass-energy equivalence; in the latter an energy-entropy equivalence of macrostates was identified within groups of similar objects. Mathematically, the former is generated from applying Pythagorean rules to the coordinates of a 4D Lorentzian geometry (two sums and a difference between four positive terms: $\left.\mathrm{d} x_{1}^{2}+\mathrm{d} x_{2}^{2}+\mathrm{d} x_{3}^{2}-\mathrm{c}^{2} \cdot \mathrm{d} t^{2}\right)$. The latter originates from correlating and partitioning energy contributions 


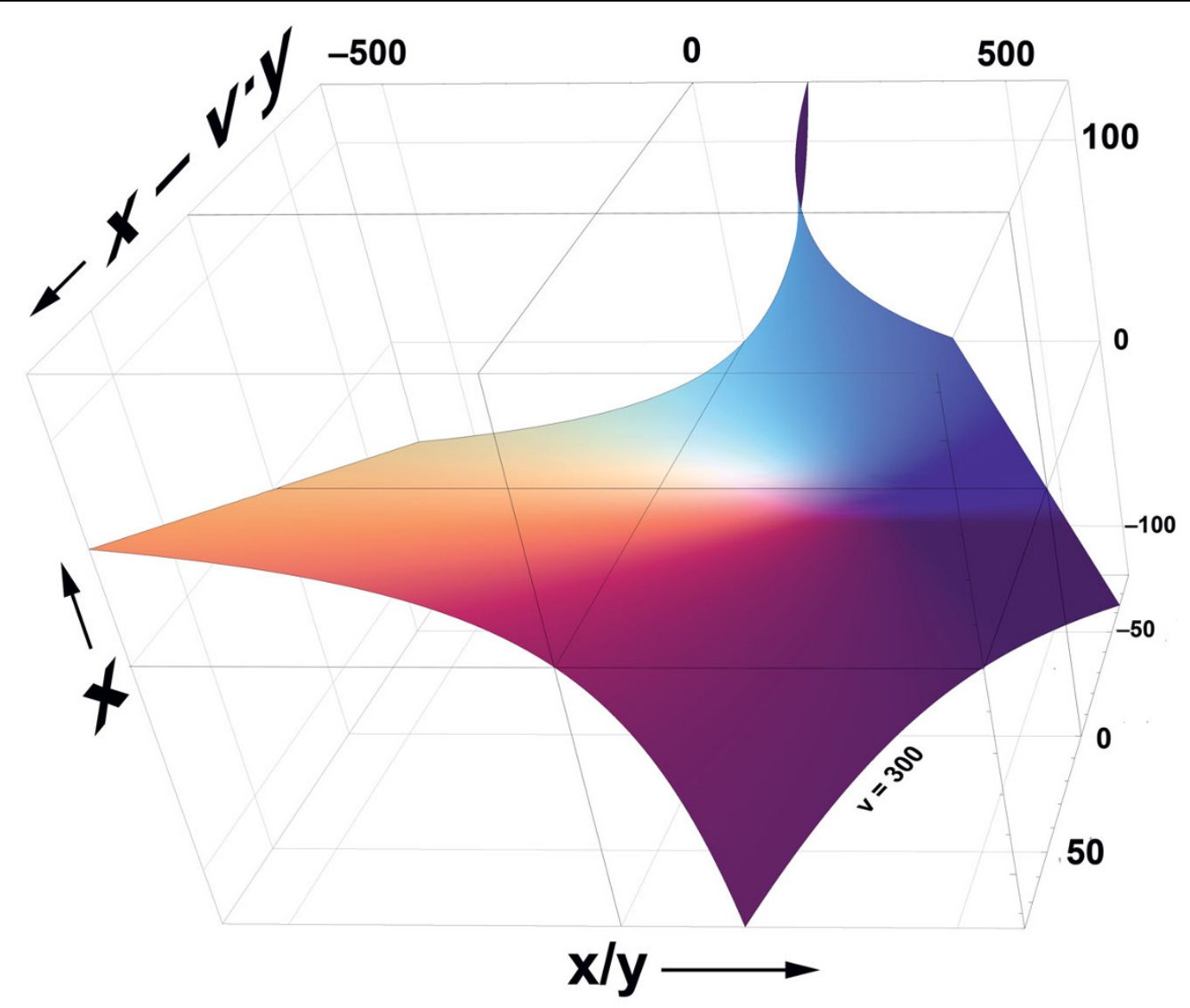

Figure $13 \mathrm{D}$ view of the relationship between difference and ratio. Equation 2 at $v=300$. The saddle point of this hyperbolic paraboloid is $\{u=x-v \cdot y=0, x=0, x / y=300\}$.

through Legendre transformations. In spite of this apparent disparity, the mathematical formalism looks alike for both theories. Lorentzian geometry and the Gibbs-Helmholtz equation are both typified through a crucial substraction term; through the mathematical difference viz. the physical balance between two most fundamental components of spacetime and, respectively, free energy. What is the squared spatial distance differential in special relativity, $\mathrm{d} x_{\mathrm{i}}{ }^{2}$, is formally the energy differential in thermodynamics, $\mathrm{d} U$ (or $\mathrm{d} H$ at constant pressure). The spacetime differential that describes changes in time not space, $\mathrm{c}^{2} \cdot \mathrm{d} t^{2}$, formally becomes the entropic component of the free energy differential, $T \cdot \mathrm{d} S$. The balance between each pair of components gives rise to the squared spatio-temporal distance differential $\mathrm{d} s^{2}$ and, respectively, the free energy differential $\mathrm{d} F$ (or $\mathrm{d} G$ at constant pressure).

\section{Inherently irreversible (de facto not reversed) versus essentially reversible dynamics}

What comes next is in part intuition rather than firm calculation all the way through. The starting point is the assumption, unproven as it is, that the energyentropy relationship for an ensemble of similar objects [1] applies to the energetics of all physical changes irrespective of scale and material; actually, irrespective of whether it is applied to normal matter, dark matter, radiation or the fabric of spacetime itself. The basis stems from mathematics; more precisely, from the relationship between difference and ratio of entities that are quantifying fundamental states of similar objects in the universe. Consider the fact that the universe, as we know it now, took its beginnings with a Big Bang, meaning that all of a sudden its total volume began to grow at rates that would vary. This changeable growth rate is usually seen as varying with time; here we shall prefer to see, both, growth rate and time varying with the universe's total volume. It grows for the simple reason that it can access to more spacetime through a not yet fully understood expansion mechanism being fueled by a dynamically biased balance between attractive force fields of normal and dark matter on the one hand, and repulsive force fields of spacetime on the other. 


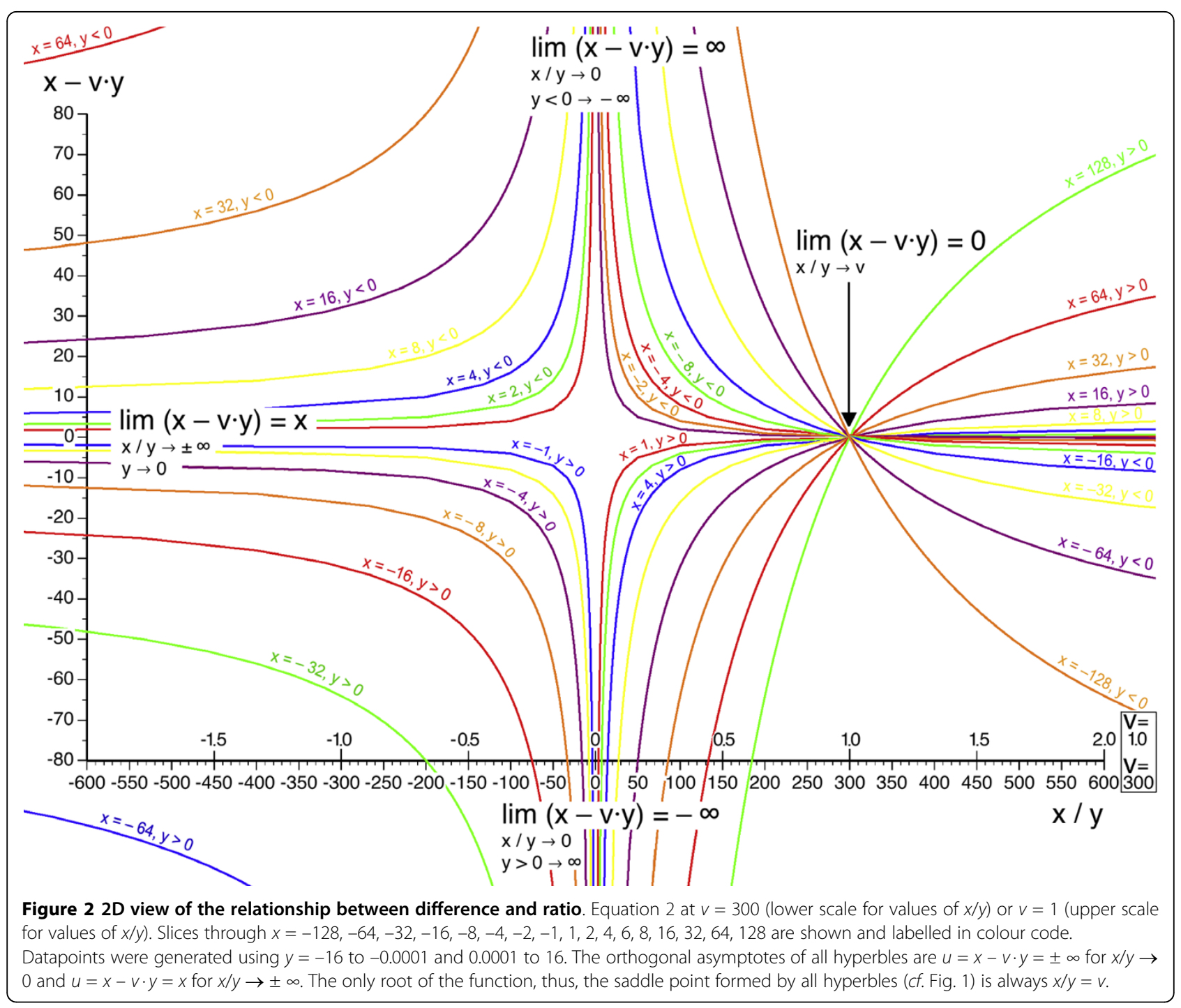

Prior to this expansion, the total of existing spacetime must have been in a minimum entropy state - or else the stringency of the Second Law could not apply - and must have accessed a minimum volume, the Planck volume. Deeper insight into the universe's 'fabric of quantum spacetime' could emerge from the description of its dynamics through the spontaneous and continuous replication and disappearance of a fundamental elementary unit of spacetime that could generate and harbour, among other 'things', matter and that would evolve in complexity through a Darwinian-like process [2-4]. A promising approach to a theory of quantum gravity is the causal dynamic triangulation (CDT) of 4D Lorentzian spacetime geometry, in which piece wise flat geometries ('4D-triangles' called four-simplices) are being proposed as fundamental elementary units of spacetime that assemble and create "discretised curvatures" [5].
The universe, at any of its states, can be globally characterised through the state variables time of cosmic expansion, call it cosmological or absolute time $t$, and average absolute temperature $T$. What is viewed as an 'independent variable' in thermodynamics, absolute temperature $T$, finds its alter ego in general relativity: the total volume $V$ of a universe. Thus, $V$ becomes the mathematically independent variable. Absolute temperature $T$ is seen as a measure for energy density that manifests itself in reversible stochastic 'undirected' movements of the constituents (a generalised 'Brownian motion'), thus, is a measure for kinetic energy density of the reversible. Integration of $T$ over all spacetime results in its total volume-dependent value $T_{\mathrm{V}}$. In this context $T_{\mathrm{V}}$ might be expressed in units of the cosmological equation of state of a perfect fluid, $\left[\mathrm{m}^{2} \cdot \mathrm{K} \cdot \mathrm{s}^{-2} \cdot \mathrm{J}^{-1}\right]$, thus, that of the squared characteristic thermal speed of 
particles, or of elementary units of spacetime, scaled by the universal gas constant $R$.

Cosmological absolute time $t$ [seconds] is also viewed as an energy density, yet manifests itself in the abundance of de facto not reversed 'directed' motion, hence, is a measure for kinetic energy density of the irreversible. Time only exists if there is change, most generally, change in the state of the object(s) in question; objects being particles or elementary units of spacetime. In a universe small enough to allow absolutely all movements to be strictly reversible, and de facto 'constantly' reversed, there is no way of measuring nor defining time. In such a situation time does not exist within the accessed volume. As soon as the first object moves in a first unreversed direction, or the first elementary unit of spacetime replicates itself without disappearance of the replica, a de facto unreversed motion materialises due to the universe's growing size. Time begins to exist (to bear a physical meaning) and grows older with the accomplished distance multiplied by the number of objects performing such 'directed' movements.

Both state variables $t$ and $T$, being measures for different aspects of energy density, are system-immanent, are not measured by an external inertial observer but are defined from within, and so could be applied in a background-independent and nonperturbative theory of quantum gravity [3-5].

"More and more, I have the feeling that quantum theory and general relativity are both deeply wrong about the nature of time. It is not enough to combine them. There is a deeper problem, perhaps going back to the origin of physics. Around the beginning of the seventeenth century, Descartes and Galileo both made a most wonderful discovery: You could draw a graph with one axis being space and the other being time. [...] In this way, time is represented as if it were another dimension of space. Motion is frozen, and a whole history of constant motion and change is presented to us as something static and unchanging. If I had to guess (and guessing is what I do for a living), this is the scene of the crime. We have to find a way to unfreeze time - to represent time without turning it into space”. Lee Smolin, 2006 [6]

\section{Time unfrozen}

One must allow the universe to develop inhomogeneously - at least the matter within, to some minimal extent even at its early stages. Absolute time $t$ is therefore expected to grow older inhomogeneously within the accessed total volume $V$, thus, to be best described by a specific energy density tensor of space. Let us derive absolute time $t$, being the de facto irreversible 'fraction' of the universe, from its entanglement with temperature at a given total volume $T_{\mathrm{V}} . T_{\mathrm{V}}$ is the measure for an average energy density of the reversible 'fraction' of the universe (perhaps one should replace 'fraction' with 'aspect') and is therefore globally diminishing. It makes sense to formulate these two aspects as fundamental cosmic variables that characterise the universe's global state at every stage of its being; as 'two aspects of the same coin' (= universe); as synonyms to some extent. This is comparable to the synonymity of mass and energy where $c^{2}$ is the 'independent variable'. (As a matter of fact, $\mathrm{c}$ is the most constant 'variable' that we can think of; other universes may bear other physics owing to other numerical values for c.) It is also comparable to the synonymity of energy and entropy for similar objects where $T$ is the 'independent variable'. Notice that in both special relativity and thermodynamics the most prominent relationships are balanced by a mathematical difference between the respective fundamental state variables, $\mathrm{d} s^{2}=\mathrm{d} x_{\mathrm{i}}^{2}-\mathrm{c}^{2} \cdot \mathrm{d} t^{2}$ and, respectively, $\mathrm{d} F=\mathrm{d} U-T \cdot \mathrm{d} S$. In contrast, general relativity calls for the substantiation of a repulsive (positive) cosmological constant $\Lambda\left[\mathrm{s}^{-2}\right]$ through the linking of its most fundamental state variables in a reciprocal relationship as shown in equation 3 .

$$
t \cdot T=\mathrm{c}^{2} \cdot R^{-1} \cdot \Lambda^{-1 / 2}
$$

Another reciprocal time-temperature relationship, $t^{0.5} \cdot T \propto \mathrm{C}$, has been derived from special relativity by George Gamow [7-10], the co-founder of the RNA Tie Club. This relationship still serves as the basis for explaining nucleosynthesis phenomena during early stages of the universe. More recently Isasi obtained $t^{0.827} \cdot T \propto c$ (derived from Table four in [11]).

Owing to the isolation of any universe de definitio and the First Law, the overall energy density remains constant throughout all states of a universe. What changes, due to a changing total volume, is the ratio of its irreversible versus reversible energy densities: in an expanding universe the former grows to the cost of the latter, time $t$ grows older as temperature $T$ cools down. Several consequences and questions arise from this relationship, some of them may seem rather strange or naive. Let me ask them anyway.

\section{Questions, the first spontaneous symmetry breaking, and consequences}

While the entropy and similarity of objects in the universe grows with time, the overall temperature diminishes and gives way to structured spacetime to the cost of structured matter; 'structured' spacetime in the sense of 'de facto irreversibly formed'. Could this be the basis - seemingly 
paradoxically - for the spontaneous non-ergodic growth of irreversibly formed 'structure', which may be read out as 'information', thus, the basis for a spontaneously increasing information density in spite of a growing number of black holes (maximum entropy objects) that are expected to evaporate at ever increasing rates?

Secondly, the limiting case for the cosmic beginning, that is, for a vanishing total volume, is described in equation 4 .

$$
\lim _{V \rightarrow 0} t=T^{-1}
$$

Within an extremely small total volume (close to the Planck volume) all movements of all particles, or the formation and vanishing of spacetime elements, are exactly reversible. There is no way at all for any change to manifest itself at any given (extremely small) $V$, and so time and temperature are exactly equivalent (eqn. 4). The spontaneous symmetry breaking - most likely the first of all - happens when $V$ becomes such that the first precise back movement is too improbable to occur. At such a critical $V$, time becomes first manifest through not reversed particle movements, or not reversed spacetime formation, and thus becomes distinguishable from reciprocal temperature.

Backwards in time: As $V$ approaches the Planckian scale, the universe's degeneracy diminishes and its temperature grows at a hyperbolically increasing rate. Only a non-zero total volume prevents the (weak) singularity of infinite absolute temperature. The existence of a first non-zero dimensional spacetime element would mean that a finite maximal temperature (= reversible energy density) characterises an 'unborn' universe in which time cannot be defined from within but also cannot be zero. How does this relate to the Planck time or the chronon? Does it mean that in the beginning, viz. on a Planckian scale for the total volume, there is a superposition of many finite entangled (very short) times that decohere with the first 'directed' motion? If yes, how many? Is there a finite population (= probability density) of very short times (= irreversible energy densities) of opposite signs averaging to nil?

Thirdly, in the formal analogy between special relativity and thermodynamics the time component of spacetime compares to the entropy component of free energy: $\mathrm{c}^{2} \cdot \mathrm{d} t^{2} \propto T \cdot \mathrm{d} S$. For a vanishing total volume, as proposed above, temperature may be replaced with reciprocal time: $T_{V \rightarrow 0}=1 / t$. It follows that $\mathrm{d} S_{V \rightarrow 0} / \mathrm{d} t \propto \mathrm{c}^{2} \cdot t \cdot \mathrm{d} t$ which integrates over $\mathrm{d} t$ into $S_{V \rightarrow 0} \propto \mathrm{c}^{2} \cdot t^{2} / 2$. For a non-zero minimal $t$, is this the residual entropy of an unborn universe?
Furthermore, when, like in group thermodynamics [1], the whole universe is treated as an isolated system of similar objects, an analogous relationship of difference between $t$ and $1 / T$ should bear a physical meaning, one that formally compares to thermodynamic free energy or to trajectories on geodesics of relativistic spacetime: If $R \cdot \Lambda^{1 / 2} \cdot \mathrm{c}^{-2}=1 / T \cdot t\left[\mathrm{~J} \cdot \mathrm{s} \cdot \mathrm{K}^{-1} \cdot \mathrm{m}^{-2}\right]$ (eqn. 3), what is $\mathrm{c}^{2} \cdot R^{-1} \cdot \Lambda^{-1 / 2} \cdot T^{-1}-s^{-3} \cdot V \cdot t=[\mathrm{s}]$ ? Like for total energy density in classical general relativity, the ratio of reciprocal temperature over time, $1 / T \cdot t$, is determined by a volume-independent ratio of fundamental constants $\left(R \cdot \Lambda^{1 / 2} \cdot \mathrm{c}^{-2}\right)$. The above difference between reciprocal temperature and time consequently describes the volume-dependence of the flow of total energy density from de facto reversed into increasingly not reversed states.

It follows from the analogy to group thermodynamics that similar cosmic objects, or similar regions of spacetime elements, can be characterised through the correlation between the above difference and ratio and have the group-characteristic shape of a 5D hyperbolic paraboloid, projections of which are depicted in Figures 1 and 2. This shape bears an all-negative curvature and the part that is close to the saddle point is produced by state variables of identical sign, thus, originates from strictly positive physical constants including $\Lambda$ (eqn. 3). This hyperbolic paraboloid is reminiscent of the saddleshaped 4D space geometry of a universe with negative energy density, as described by the anti-de-Sitter space/ conformal field theory correspondence (also known as Polyakov's conjecture and Maldacena duality). Similar cosmic objects or similar regions of spacetime elements are expected to reveal a group-characterising linearity for volume-dependent energy density (= difference) versus reciprocal temperature over time (= ratio). This linearity may be best observable at a total volume of the universe in which both reversible and irreversible components of the considered objects (spacetime regions) appear balanced within the group.

Finally, useful insights might emerge from common grounds for physics seen as a difference-ratio relationship of fundamental constants, as shown here, and Alain Connes' formulation of noncommutative geometries [12] through a deep analysis of addition and multiplication groups and their recently explored connection to entropy and statistical thermodynamics [13].

The author of this 'Perspectives' article, an organic biomolecular experimental chemist with an open mind for physics and cosmology, would like to know from the physics community (see Appendix 1), which of the above conclusions and statements make sense, whether they may be experimentally testable and, if yes, how? 


\section{Appendix 1}

A very preliminary version of this manuscript was published on $15^{\text {th }}$ June 2009 [14]. On my private calendar I marked the $11^{\text {th }}$ May 2003 as the morning of inspiration on time-temperature equivalence and volume-dependent spontaneous symmetry breaking (date unproven).

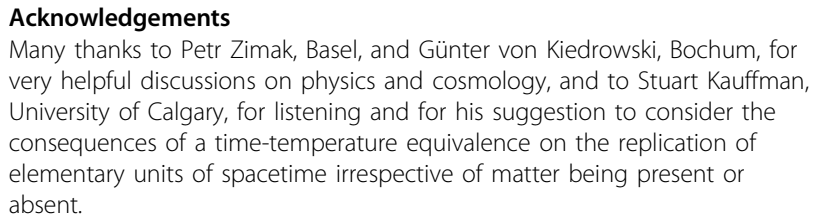

Received: 1 December 2009 Accepted: 1 September 2010

Published: 1 September 2010

\section{References}

1. Zimak P, Terenzi S, Strazewski P: New concept for quantification of similarity relates entropy and energy of objects: First and Second Law entangled, group behavior of micro black holes expected. J Syst Chem 2010, 1:2.

2. Smolin L: Did the universe evolve? Class Quant Grav 1992, 9:173-191.

3. Kauffman S, Smolin L: A possible solution for the problem of time in Quantum Cosmology. The Third Culture, Edge Foundation Inc 1997 [http:// edge.org/3rd_culture/smolin/smolin_p1.html].

4. Kauffman $\mathrm{S}$, Smolin L: Combinatorial dynamics and time in quantum gravity. In Towards Quantum Gravity, Lecture Notes in Physics. Edited by: Kowalski Glikman J. Springer, Berlin; 2000:541:101-129.

5. Ambjørn J, Jurkiewicz J, Loll R: The universe from scratch. [http://arxiv.org/ abs/hep-th/0509010]

6. Smolin L: The Trouble With Physics. Mariner Books, Boston, New York 2006, 256-257

7. Gamow G: The origin of elements and the separation of galaxies. Phys Rev 1948, 74:505-506.

8. Alpher RA, Bethe H, Gamow G: The origin of chemical elements. Phys Rev 1948, 73:803-804.

9. Gamow G: Expanding universe and the origin of elements. Phys Rev 1946, 70:572-573.

10. Gamow G: Erratum: Expanding universe and the origin of elements. Phys Rev 1947, 71:273.

11. Isasi RA: Equivalence between the empty microspace and the cosmological space. [http://vixra.org/abs/0909.0015].

12. Connes A: Noncommutative Geometry. Academic Press, San Diego, CA 1994, ISBN 0-12-185860-X.

13. Connes A, Consani C: Characteristic 1, entropy and the absolute point. [http://alainconnes.org/en/downloads.php].

14. Zimak P, Terenzi S, Strazewski P: New concept for quantification of similarity relates entropy and energy of objects: First and Second Law entangled, equivalence of temperature and time proposed. [http://arxiv. org/abs/0906.2799].

doi:10.1186/1759-2208-1-11

Cite this article as: Strazewski: The relationship between difference and ratio and a proposal: Equivalence of temperature and time, and the first spontaneous symmetry breaking. Journal of Systems Chemistry 2010 1:11.

\section{Publish with ChemistryCentral and every scientist can read your work free of charge \\ "Open access provides opportunities to our colleagues in other parts of the globe, by allowing anyone to view the content free of charge." W. Jeffery Hurst, The Hershey Company. \\ - available free of charge to the entire scientific community \\ - peer reviewed and published immediately upon acceptance \\ - cited in PubMed and archived on PubMed Central \\ - yours - you keep the copyright \\ Submit your manuscript here: \\ http://www.chemistrycentral.com/manuscript/<smiles>c1ccccc1</smiles> Chemistry Central}

